recanalization, 24- hour follow up imaging, and mRS at 90 days.

Result In these 29 patients, they were 14 were males, 68 years old (42-81 years old). The initial NIHSS at emergency department was 27 (standard deviation $(\mathrm{SD})=7.3$ ); 8 of them received IV-tPA, the average time from onset to needle was 417 minutes ( $\mathrm{SD}=38.9)$; 93.1\% (27/29) using aspiration as first method of recanalization; TICI $=3$ or $2 \mathrm{~b}$ in $86.2 \%(25 /$ $29)$; 41.4\% (12/29) associated with basilar artery stenosis; on the 24-hour follow up imaging, parenchymal hemorrhage was found in 3 and hemorrhagic transformation in 3; the mRS $(0-2)$ at 90 days was $55.1 \%(16 / 29)$, and the mortality rate was $10.3 \%(3 / 29)$.

Conclusion Acute basilar artery occlusion is a complex and severe disease, endovascular thrombectomy can have a high rate of recanalization but the clinical outcome on 90 days still worse than the one in anterior circulation. The treatment will become more challenge in patient associated with underlying intracranial atherosclerotic stenosis.

Disclosures H. Liu: 3; C; Stryker, GE. C. Lee: None. Y. Lin: None. C. Wan: None.

\section{INTRA-ARTERIAL THROMBOLYSIS AS RESCUE THERAPY IN MCA OCCLUSIONS: SUBANALYSIS FROM THE STRATIS REGISTRY}

${ }^{1} \mathrm{~A}$ Castonguay*, 'S Zaidi, 'M Jumaa, ${ }^{2} \mathrm{O}$ Zaidat, ${ }^{3} \mathrm{D}$ Liebeskind, ${ }^{1} \mathrm{H}$ Salahuddin, ${ }^{4} \mathrm{~N}$ MuellerKronast. 'University of Toledo, Toledo, OH; ${ }^{2}$ Mercy St. Vincent, Toledo, OH; ${ }^{3} \mathrm{UCLA}$, Los Angeles, CA; ${ }^{4}$ Delray Medical Center, Delray Beach, FL

\subsection{6/neurintsurg-2019-SNIS.231}

Background and purpose Recent data suggests that intra-arterial (IA) thrombolytics may be a safe rescue therapy (RT) for acute ischemic stroke (AIS) patients after failed mechanical thrombectomy (MT); however, its safety and efficacy in MCA occlusions remains unclear. Here, we investigate the use of IA tissue plasminogen activator (IA-rtPA) as RT in MCA occlusions in the Systematic Evaluation of Patients Treated With Neurothrombectomy Devices for Acute Ischemic Stroke (STRATIS) Registry.

Methods The STRATIS Registry was a prospective, multicenter, non-randomized, observational study of AIS LVO patients treated with the Solitaire stent-retriever as the first choice therapy within 8 hours from symptoms onset. Clinical and angiographic outcomes were compared in patients with MCA occlusions treated with and without IA-rtPA.

Results A total of $680 / 938$ (72.5\%) patients with IA-tPA use harbored MCA occlusions, of which 585 (86.0\%) and 95 $(14.0 \%)$ were in the no IA-rtPA and IA-rtPA groups, respectively. Baseline demographics were well-balanced between the cohorts. The majority of MCA occlusions were located in the M1 segment, with $76.1 \%$ in the no IA-rtPA group and $74.7 \%$ in the IA-rtPA group $(\mathrm{p}=0.80)$. IV-rtPA was administered in $64.4 \%$ and $71.6 \%$ of the no IA-rtPA and IA-rtPA patients $(p=0.20)$. Median IA-rtPA dose was 4mg (IQR 2$10)$ in the IA-rtPA cohort. Onset to puncture time was less than 6 hours in $88.6 \%(597 / 674)$ of patients. Mean onset to arterial puncture time was shorter in the IA-rtPA group (196.7 \pm 109.9 versus 228.2 \pm 97.9 minutes, $p=0.004)$; however, mean puncture to procedure end time was longer in the IA-rtPA group than the no IA-rtPA group $(74.5 \pm 41.5$ versus $59.5 \pm 33.6$ minutes). Mean number of passes $(2.0 \pm 1.2$ versus $1.6 \pm 1.0, \mathrm{p}=0.005)$ and rate of distal embolization
(53.4\% versus $72.1 \%, p=0.001)$ were significantly higher in the IA-rtPA group. The rate of substantial reperfusion $(\mathrm{mTICI} \geq 2 \mathrm{~b})$ was similar in the cohorts $(89.9 \%$ versus $86.0 \%, \mathrm{p}=0.28)$. Rates of symptomatic intracranial hemorrhage $(\mathrm{sICH})(1.7 \%$ versus $2.2 \%, \mathrm{p}=0.68)$, good functional outcome (mRS $\geq 2,60.5 \%$ versus $58.0 \%, \mathrm{p}=0.85$ ), and mortality $(13.9 \%$ versus $13.6 \%, p=0.96)$ at 90 days did not differ between the two groups.

Conclusion Use of IA-rtPA as rescue therapy after failed mechanical thrombectomy in MCA occlusions was not associated with an increased risk of sICH or mortality in the STRATIS Registry. More studies are needed to understand the safety and efficacy of IA thrombolysis as rescue therapy in this patient population.

Disclosures A. Castonguay: None. S. Zaidi: None. M. Jumaa: None. O. Zaidat: None. D. Liebeskind: None. H. Salahuddin: None. N. Mueller-Kronast: None.

\section{FACTORS ASSOCIATED WITH THE DECISION-MAKING ON ENDOVASCULAR THROMBECTOMY FOR FHE MANAGEMENT OF ACUTE ISCHEMIC STROKE}

${ }^{1} \mathrm{M}$ Goyal ${ }^{*},{ }^{2} \mathrm{~B}$ Menon, ${ }^{3} \mathrm{~N}$ Kashani, ${ }^{3} \mathrm{~A}$ Wilson, ${ }^{4} \mathrm{~S}$ Yoshimura, ${ }^{5} \mathrm{~B}$ Campbell, ${ }^{6} \mathrm{~B}$ Baxter, ${ }^{7} \mathrm{~A}$ Rabinstein, ${ }^{8} \mathrm{~F}$ Turjman, ${ }^{9} \mathrm{U}$ Fischer, ${ }^{10} \mathrm{~J}$ Ospel, ${ }^{11} \mathrm{~W}$ Kunz, ${ }^{12} \mathrm{P}$ Mitchell, ${ }^{13} \mathrm{P}$ Sylaia, ${ }^{14} \mathrm{M}$ Cherian, ${ }^{15} \mathrm{~B}$ Kim, ${ }^{16} \mathrm{~J} \mathrm{Heo},{ }^{17} \mathrm{~A}$ Podlasek, ${ }^{3} \mathrm{M}$ Almekhlafi, ${ }^{3} \mathrm{M}$ Foss, ${ }^{18} \mathrm{~A}$ Demchuk, ${ }^{18} \mathrm{M}$ Hill, ${ }^{19} \mathrm{G}$ Saposnik. ${ }^{1}$ Radiology, Clinical Neurosciences, University of Calgary, Calgary, $A B$, Canada; ${ }^{2}$ Clinical Neurosciences, University of Calgary, Calgary, $A B$, Canada; ${ }^{3}$ Radiology, University of Calgary, Calgary, $A B$, Canada; ${ }^{4}$ Neurosurgery, Hyogo College of Medicine, Hyogo, Japan; ${ }^{5}$ Neurology, Royal Melbourne Hospital, University of Melbourne, Melbourne, Australia; ${ }^{6}$ Radiology, Erlanger Hospital Chattanooga, Chattanooga, TN; ${ }^{7}$ Radiology, Mayo Clinic Rochester, Rochester, MN; ${ }^{8}$ Radiology, Centre Hospitalier Universitaire de Lyon, Lyon, France; ${ }^{9}$ Radiology, University Hospital Bern (Inselspital), Bern, Switzerland; ${ }^{10}$ Radiology, University Hospital Basel, Basel, Switzerland; ${ }^{11}$ Radiology, University Hospital Munich, Munich, Germany; ${ }^{12}$ Radiology, Royal Melbourne Hospital, University of Melbourne, Melbourne, Australia; ${ }^{13}$ Radiology, Sree Chitra Tirunal Institute for Medical Sciences and Technology, Thiruvananthapuram, India; ${ }^{14}$ Radiology, Kovai Medical center, Tamil Nadu, India; ${ }^{15}$ Radiology, St. Mary's Hospital Seoul, Seoul, Korea, Republic of; ${ }^{16}$ Neurology, Yonsei University, Seoul, Seoul, Korea, Republic of; ${ }^{17}$ Radiology, Southend University Hospital NHS Foundation Trust, Westcliff-on-Sea, UK; ${ }^{18}$ Neurology, University of Calgary, Calgary, AB, Canada; ${ }^{19}$ Neurology, St. Michael's hospital, Toronto, ON, Canada

\subsection{6/neurintsurg-2019-SNIS.232}

Background and Purpose Little is known about the real-life factors that clinicians use in selection of patients that would receive endovascular treatment (EVT) in the real world. We sought to determine patient, practitioner, and health system factors associated with therapeutic decisions around endovascular treatment.

Methods We conducted a multinational cross-sectional webbased study comprising of 607 clinicians and interventionalists from 38 countries who are directly involved acute stroke care. Participants were randomly allocated to 10 from a pool of 22 acute stroke case scenarios and stated how they would treat the patient A) under current local resources, and B) under assumed ideal conditions (i.e. without monetary or infrastructural constraints). Descriptive statistics and logistic regression was performed to determine multilevel factors associated with EVT, adherence to current EVT guidelines, and practice gaps between current local resources and assumed ideal conditions. Results Of the 1330 invited participants, 607 (45.6\%) participants completed the study $(53.7 \%$ neurologists, $28.5 \%$ neurointerventional radiologists, $17.8 \%$ other clinicians). NIHSS (34.9\%), level of evidence (30.2\%), ASPECTS or ischemic core volume (22.4\%), patient's age (21.6\%), and clinicians' 